\title{
Chalcones: A review on synthesis and pharmacological activities
}

\author{
Kamya Goyal ${ }^{1,2}$, Rajwinder Kaur ${ }^{2 *}$, Anju Goyal ${ }^{2}$, Rajendra Awasthi ${ }^{3}$ \\ ${ }^{1}$ Laureate Institute of Pharmacy, Jawalamukhi, Himachal Pradesh, India. \\ ${ }^{2}$ Chitkara College of Pharmacy, Chitkara University, Rajpura, India. \\ ${ }^{3}$ Amity Institute of Pharmacy, Amity University Uttar Pradesh, Noida, Uttar Pradesh, India.
}

\section{ARTICLE INFO \\ Received on: 30/08/2019 \\ Accepted on: 22/08/2020 \\ Available online: 25/02/2021}

Key words:

Chalcone, Claisen-Schmidt

condensation, anticancer,

antimicrobial, antidiabetic.

\begin{abstract}
Chalcone is a privileged species with medicinal significance as it consists of reactive ketoethylenic moiety - $\mathrm{CO}-$ $\mathrm{CH}=\mathrm{CH}$ - belonging to flavonoids. The presence of a reactive $\alpha, \beta$-unsaturated carbonyl function in chalcone and its derivatives is the reason for its pharmacological activities. Chalcones exhibit a wide spectrum of pharmacological effects such as antioxidant, antibacterial, anthelmintic, antiulcer, antiviral, insecticidal, antiprotozoal, anticancer, antiinflammatory, antidiabetic, etc. Chalcones can be synthesized by Claisen-Schmidt's condensation, Heck's reaction, Suzuki's reaction, etc. The purpose of this review is to focus on the methods of synthesis of chalcones and their versatile pharmacological activities.
\end{abstract}

\section{INTRODUCTION}

The chemistry of chalcone PGs is gaining intense research interest globally. The term "Chalcone" was coined by Kostanecki and Tambor (1899). Other names for chalcones are benzyl acetophenone or benzylideneacetophenone. In the structure of a chalcone, two benzenoid rings are joined by an aliphatic chain of three carbons. Chalcone is an $\alpha, \beta$-unsaturated ketonic compound consisting of two benzenoid rings with wide variety of groups. Aromatic groups are connected to each other by three carbons, $\alpha$, $\beta$-unsaturated ketonic system, highly electrophilic in nature having a linear structure (Awasthi et al., 2009; Cheng et al., 2000; Liu et al., 2001). They have ketoethylenic moiety $(-\mathrm{CO}-\mathrm{CH}=\mathrm{CH}-)$ in their structure. They have a conjugated double bond and an entirely delocalized $\pi$-electron-containing order on aromatic rings. Chalcones have been utilized as a precursor for the synthesis of compounds which possess pharmacological importance (Straub, 1995). The chalcones' chemistry remains a major interest for scientists in the 21 st century, producing a diversity of promising pharmacological activities like anti-inflammatory (Dhar et al.,

Abstract of this article was presented at CUDC consortium and Summer School Conference at Chitkara University.

*Corresponding Author

Rajwinder Kaur, Chitkara College of Pharmacy, Chitkara University,

Rajpura, India.E-mail: rajwinder.kaur@chitkara.edu.in
2018; Fu et al., 2019; Gan et al., 2018; Li et al., 2017; Mahapatra et al., 2017; Md Idris et al., 2018; Sayed et al., 2018), analgesic (Fu et al., 2019), antigout as xanthine oxidase inhibitors (Hofmann et al., 2016), antihistaminic (Padaratz et al., 2009; Rossi and Avellino, 1957), anticancer (Gan et al., 2018; Hsieh et al., 2019; Khanapure et al., 2018; Özdemir et al., 2017; Pingaew et al., 2014; Sashidhara et al., 2010), antileishmanial (Insuasty et al., 2015), antimalarial (Pingaew et al., 2014), antiviral (Wan et al., 2015), antiulcer (Choudhary et al., 2012), antimicrobial (Benouda et al., 2019; Lal et al., 2018; Monga et al., 2014; Özdemir et al., 2017; Sayed et al., 2018), antioxidant (Bandgar et al., 2010), antidiabetic (Balu et al., 2019; Emayavaramban et al., 2013; Gaur et al., 2014; Hsieh et al., 2012; Rammohan et al., 2020; Shukla et al., 2017), etc. Metochalcones increase bile secretion by stimulating the liver (Sahu et al., 2012) and sofalcone as an antiulcer agent, which increases the concentration of Prostaglandins from the mucosa causing a gastroprotection from Helicobacter pylori-induced ulcers (Higuchi et al., 2010). It is also found through clinical trials that hesperidin methylchalcone was tested and found effective for chronic peripheral venous lymphatic insufficiency (Beltramino et al., 1999, 2000) and hesperidin trimethylchalcone was found effective for trunk or branch varicosis (Weindorf and SchultzEhrenburg, 1987). 
<smiles>COc1ccc(/C=C/C(=O)c2ccc(OC)cc2OC)cc1</smiles><smiles>[R]C(=O)Oc1cc(/C=C/C(=O)c2c(O)cc(OC3O[C@H](CO[C@H]4OC([M])[C@H](O)C(O)[C@H]4O)[C@@H](O)C(O)[C@H]3O)cc2O)ccc1OC</smiles>

Hesperidin methylchal cone $\mathrm{R}=\mathrm{H}$ Hesperidin trimethylchal cone $\mathrm{R}=\mathrm{CH}_{3}$

Chalcones have been known from earlier times to have an interesting moiety which is associated with a wide range of pharmacological activities. The majority of commonly found food chalcones are phloretin (Gerhauser, 2008; Mariadoss et al., 2019; Min et al., 2015) and its glucoside phloridzin, i.e., phloretin 2'-0$\beta$ - glucopyranoside which are present in apples, chalconaringenin in tomatoes (Echeverria et al., 2009; Kolot et al., 2019; Slimestad and Verheul, 2011), Arbutin in pears (Reiland and Slavin, 2015; Sasaki et al., 2014), and flavokavains in kava plants (Liu et al., 2018; Pinner et al., 2016). Chalcone possess a very good moiety due to which a variety of novel heterocyclic compounds with better pharmacological properties can be designed.<smiles>O=C(/C=C/c1ccccc1)c1ccccc1</smiles>

Structure of a chalcone

\section{METHODS OF SYNTHESIS OF CHALCONES}

Chalcones possess a simple moiety which makes its substitutions easy with simple and easy methods of synthesis. Currently, a wide range of schemes are available to synthesize various chalcone analogs.

\section{Claisen-Schmidt's condensation}

It is a commonly employed and easy method (Scheme 1). In the method, chalcones are synthesize by condensing substituted or unsubstituted benzaldehyde with substituted or unsubstituted acetophenone with the use of bases or acids as catalysts in an appropriate solvent at about $50^{\circ} \mathrm{C}-100^{\circ} \mathrm{C}$ for few hours (Kaur and Narasimhan, 2018; Khanapure et al., 2018; Monga et al., 2014; Özdemir et al., 2017; Rahman et al., 2007; Reddy and Kathale, 2018). It is normally carried out in the liquid phase, but some syntheses occur in the solid phase, like resin was bound with

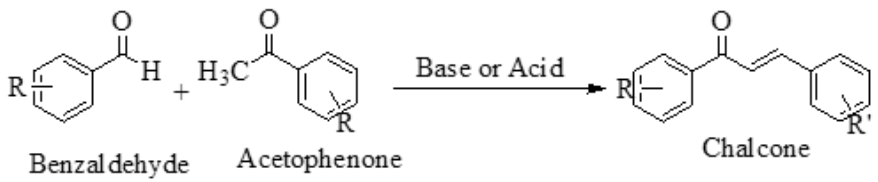

Scheme 1. The Claisen-Schmidt condensation. acetophenone compounds and then reacted with benzaldehyde compounds (Mahapatra et al., 2015) or under solvent-free conditions such as catalytic condensation in the presence of triazabicyclodecene (Fringuelli et al., 2004). Additionally, microwave-assisted liquid and solvent-free condensation decrease synthesis time and elevate the production yield (Kakati and Sarma, 2011; Srivastava, 2008).

\section{Carbonylative Heck's coupling reaction}

Chalcones have been synthesized by vinylation of aryl halide (such as phenyl halide) with styrene under carbon monoxide and the catalyst palladium can undergo carbonylative coupling (Bianco et al., 2003; Wu et al., 2010) (Scheme 2).

\section{Suzuki-Miyaura's coupling reaction}

This coupling reaction takes place by combining benzoyl chloride and styryl boronic acid using $\mathrm{Pd}\left(\mathrm{PPh}_{3}\right)_{4}, \mathrm{CsCO}_{3}$, and anhydrous toluene or by combining phenyl boronic acid and cinnamoyl chloride using $\mathrm{Pd}\left(\mathrm{PPh}_{3}\right)_{4}, \mathrm{CsCO}_{3}$, and anhydrous toluene (Selepe and Van Heerden, 2013) (Scheme 3).

\section{Sonogashira's isomerization coupling}

This reaction involves the synthesis of chalcones by the microwave coupling of the electron-insufficient group, like phenyl halide, and prop-2-yn-1-ol and catalyst $\mathrm{PdCl}_{2}\left(\mathrm{PPh}_{3}\right)_{2}$ and solvent like tetrahydrofuran (THF) (Braun et al., 2006; Takahashi et al., 1980) (Scheme 4).

\section{Continuous-flow deuteration reaction}

Ynones basically were synthesized by the process available in the literature by the reaction of benzoyl chloride and phenylacetylene under Sonogashira's conditions and then for deuteration, which was carried out in an H-Cube system caused by replacing $\mathrm{H}_{2} \mathrm{O}$ with $\mathrm{D}_{2} \mathrm{O}$ as the deuterated source (Hsieh et al., 2015; Ötvös et al., 2016) (Scheme 5).

\section{Solid acid catalyst-mediated reaction}

Chalcones are prepared by using a solid acid catalyst which is heterogeneous in nature (Scheme 6). It involves the addition of aromatic aldehyde (such as benzaldehyde) and ethynyl benzene in ethylene dichloride solvent using a microwave condition and using ion-exchange resin, like amberlyst-15, as the solid acid catalyst (Rueping et al., 2011)

\section{Coupling reaction}

Chalcones are prepared by coupling benzaldehyde with phenylacetylene in hydrogen bromide and ionic liquids like BmimOTs (1-butyl-3-methyl- $1 H$-imidazolium 4-methylbenzenesulfonate) for about 12 hours at $100^{\circ} \mathrm{C}$ (Xu et al., 2004) (Scheme 7).

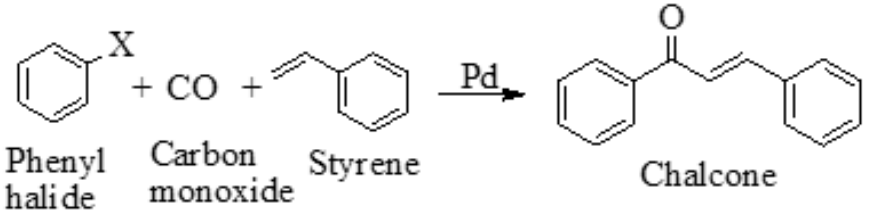



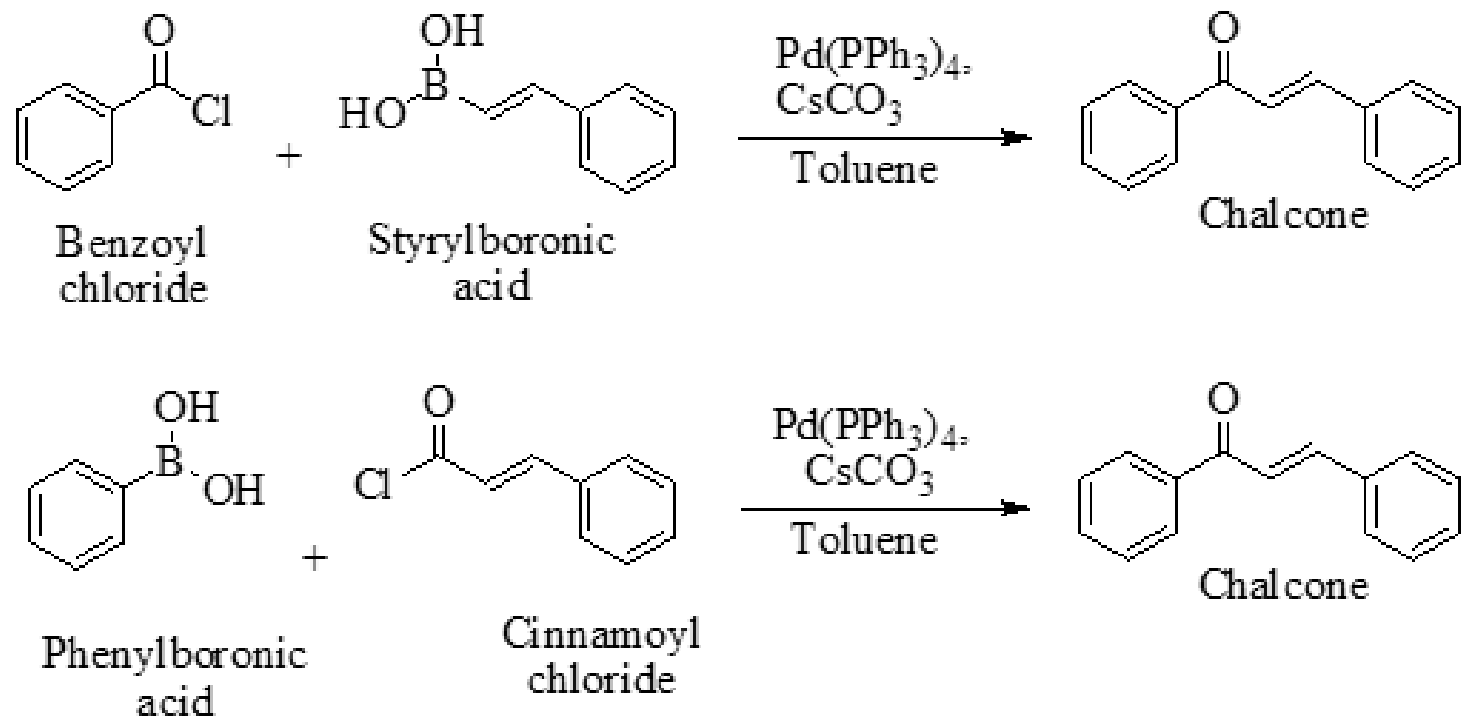

Scheme 3. Suzuki-Miyaura coupling reaction.

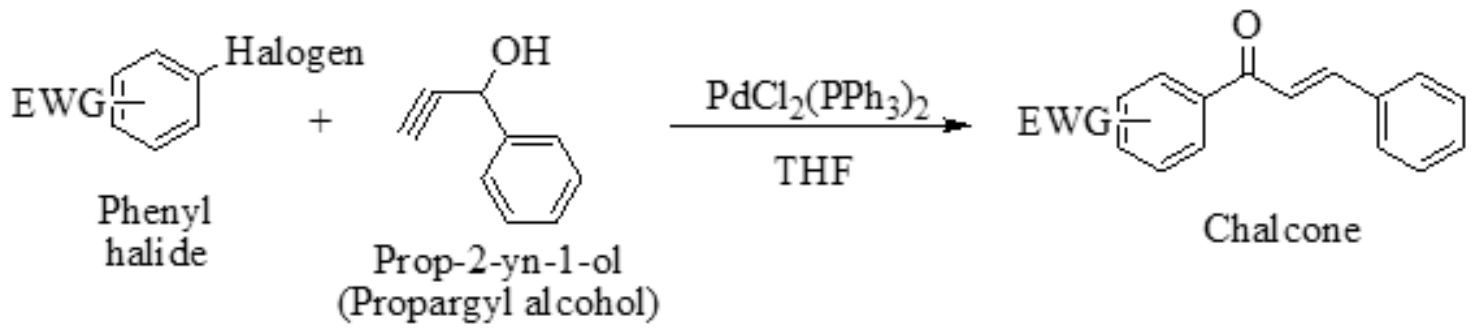

Scheme 4. Sonogashira isomerization coupling.

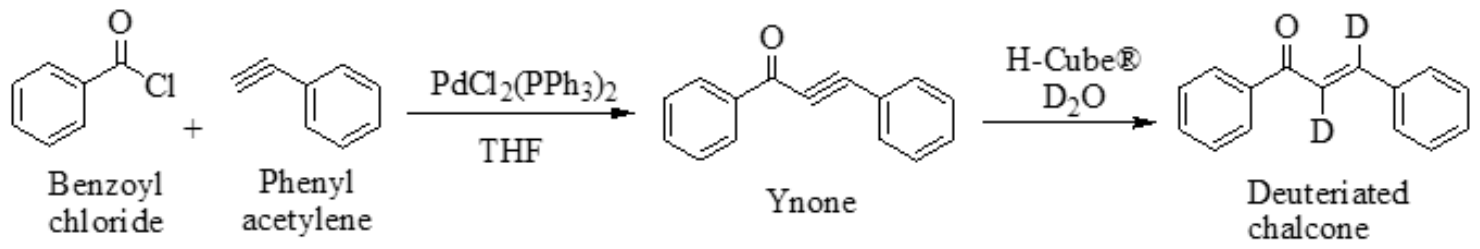

Scheme 5. Continuous-flow deuteration reaction.

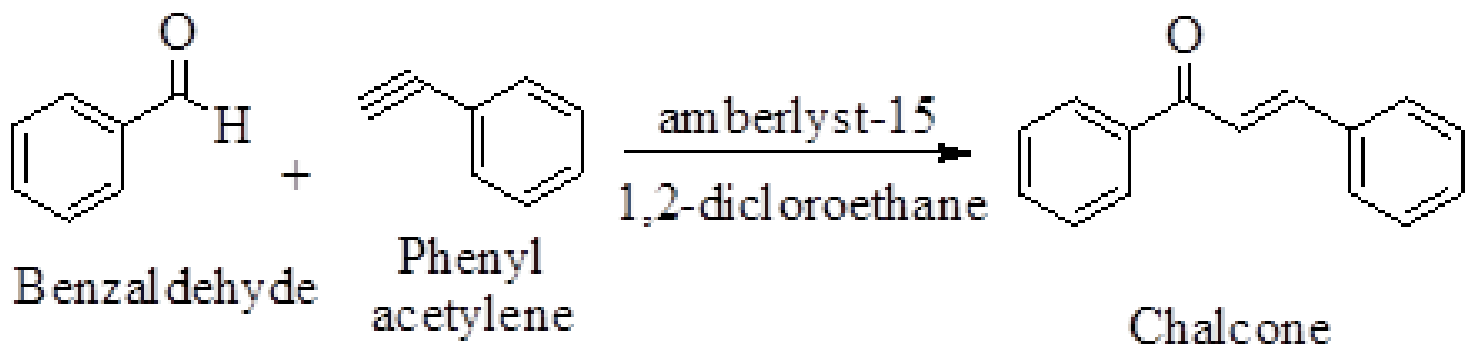

Scheme 6. Solid acid catalyst-mediated synthesis.

\section{One-pot synthesis}

It is an easy, efficient, and green method that allows the chalcone synthesis in a single reactor (Scheme 8). The reaction mixture consists of phenyl methanol and acetophenone with an oxidizing agent such as $\mathrm{CrO}_{3} \cdot \mathrm{CrO}_{3}$ generates the benzaldehyde from phenyl methanol, which then involves the reaction with the acetophenone to give chalcone (Mahapatra et al., 2015). 


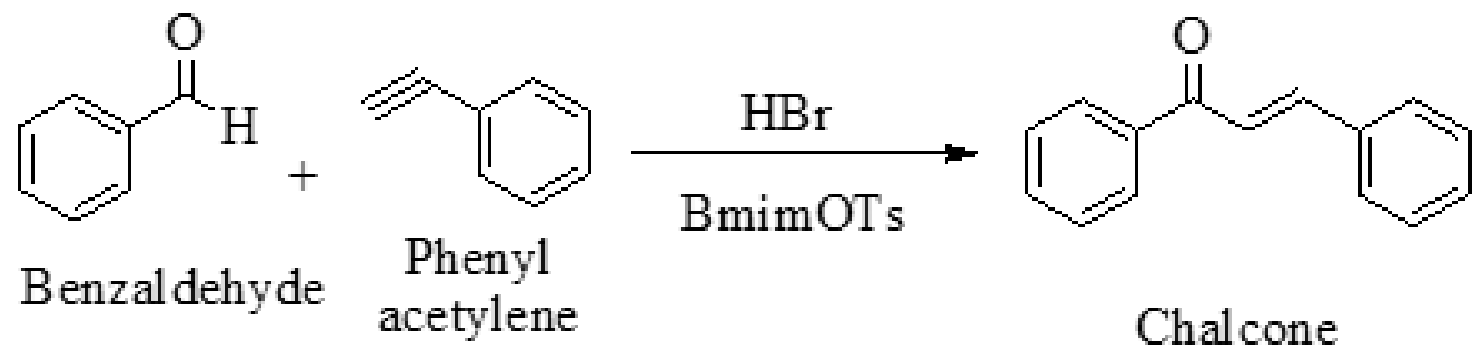

Scheme 7. Coupling reaction.<smiles>CC(=O)c1ccccc1</smiles>

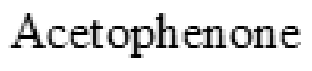

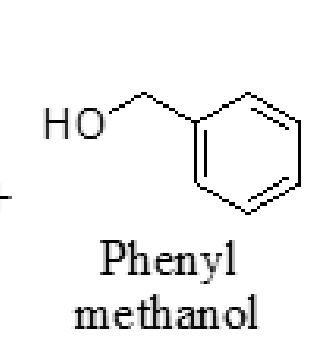<smiles>CCOCCOC(=O)c1ccccc1</smiles>

Scheme 8. One-pot synthesis of chalcones.

\section{Synthesis of chalcones using Schiff bases}

Schiff bases result in aryl amino ketones, which in the presence of an acid lead to hydramine breakdown and produce products such as primary aromatic amine and chalcones (Abe et al., 2003; Gaonkar and Vignesh, 2017).

\section{Microwave-assisted synthesis of chalcone}

In this method, heterogeneous catalysts, such as $\mathrm{K}_{2} \mathrm{CO}_{3}$, $\mathrm{Ba}(\mathrm{OH})_{2}$, p-Toluenesulfonic acid, $\mathrm{KF}-\mathrm{Al}_{2} \mathrm{O}_{3}$, piperidine, and aqueous alkali, are employed to synthesize chalcones and their derivatives under microwave conditions (Blass, 2002; Gall et al., 1999; Mistry and Desai, 2004).

\section{Ultrasound irradiation-assisted synthesis of chalcone}

Ultrasound-assisted synthesis is another advantageous technique like microwave irradiation-assisted synthesis due to the fact that it completes the reaction within short period of time and yields a high percentage of products. For the synthesis of chalcones and their derivatives under ultrasound irradiation, heterogenous catalysts like $\mathrm{K}_{2} \mathrm{CO}_{3}$, pulverized potassium hydroxide $(\mathrm{KOH})$, $\mathrm{NaOH}$, basic $\mathrm{Al}_{2} \mathrm{O}_{3}, \mathrm{KF}-\mathrm{Al}_{2} \mathrm{O}_{3}$ are used productively (Adole et al., 2020; Calvino et al., 2006; Cancio et al., 2019; Li et al., 2002; Polo et al., 2019; Rammohan et al., 2020).

\section{PHARMACOLOGICAL ACTIVITIES}

Various chalcones and their derivatives have been synthesized and reported to have pharmacological activities like antimicrobial, antimalarial, anticancer, antifungal, anthelmintic, antiinflammatory, anti-HIV, monoamine oxidase inhibition, antiangiogenic, antileishmanial activities, etc. A brief outline of some of the selected pharmacological activities is presented in the following sections.

\section{Chalcone as an antimicrobial agent}

$\alpha, \beta$-unsaturated keto functions as highly reactive species, which shows nucleophilic conjugate addition of important protein due to which it shows antimicrobial activity.

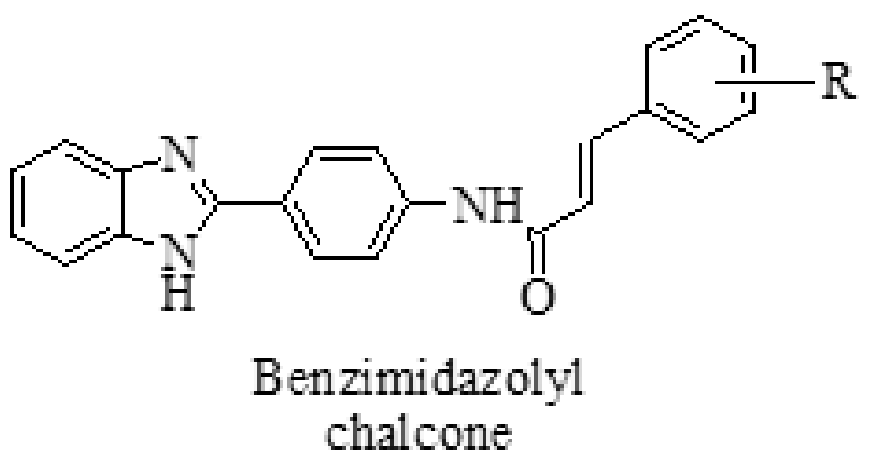

The synthesis of a new chalcone series with benzimidazolyl group was carried out to produce antimicrobial agents by condensing $\mathrm{N}-(4-(1 H$ benzo[d]imidazol-2-yl)phenyl) acetamide with benzaldehyde-related compounds using aqueous $\mathrm{KOH}$ at room temperature (Baviskar et al., 2009).

Novel nitrochalcones were synthesized to produce antimicrobial agents by condensing nitroacetophenone with some aromatic aldehydes by using a base at room temperature (Monga et al., 2014).

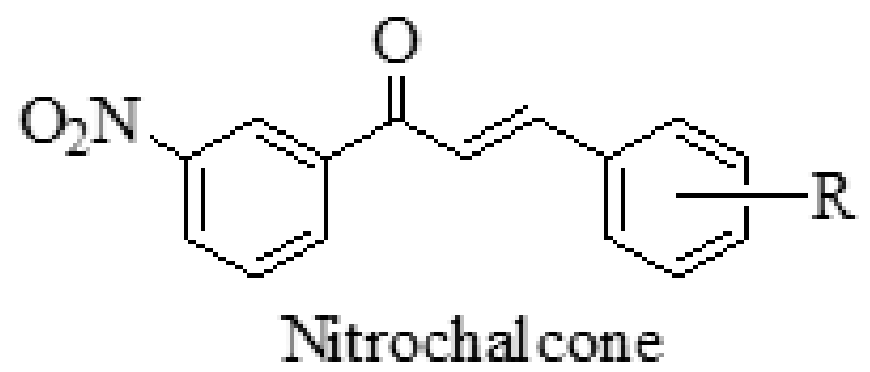

Chalcones AA(1-6) were prepared by Claisen-Schmidt's condensation of 2-acetyl pyridine and aldehyde derivatives in diluted ethanolic $\mathrm{KOH}$ at room temperature (Prasad et al., 2008). 
<smiles>[R]C=CC(=O)c1ccccn1</smiles>

AA (1-6)

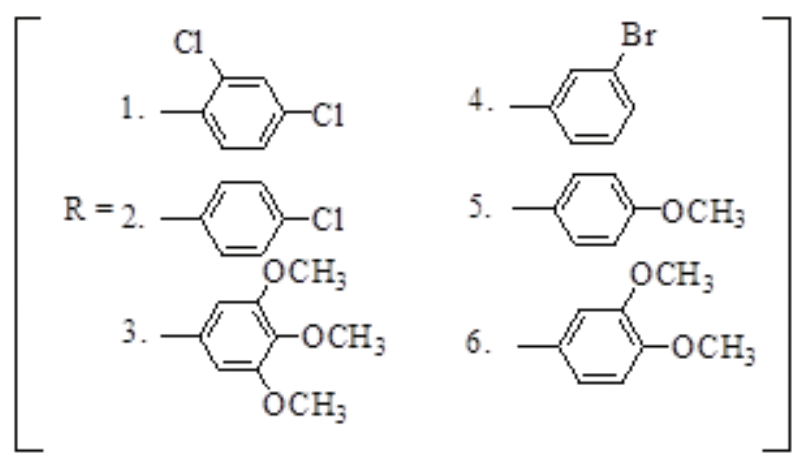

Physical properties of chalcones are presented in Table 1. Chalcones $\mathrm{AB}$ are synthesized by condensing aldehydes with o-hydroxyl acetophenone, followed by reaction with $\mathrm{I}_{2}$ and dimethyl sulfoxide (DMSO), which results in the synthesis of flavones and which shows antimicrobial property (Rathore et al., 2015).<smiles>CC(=O)c1cc(Cl)ccc1O</smiles><smiles>[R]c1ccc(C=O)c(C(=O)O[Na])c1</smiles><smiles>[R]c1cccc(/C=C\C(=O)c2cc(Cl)ccc2O)c1</smiles><smiles>[R]c1cccc(-c2cc(=O)c3cc(Cl)ccc3o2)c1</smiles>

$\mathrm{AB}$

$\mathbf{R}=\mathrm{H}, \mathrm{OH}, \mathrm{Cl}, \mathrm{NO}_{2}$

Some of the novel fluorinated chalcones, AC, AD, AE (1-13), have been synthesized and tested for antitubercular activity for Mycobacterium tuberculosis $\mathrm{H} 37 \mathrm{Rv}$ and antimicrobial activity for fungi and pathogenic bacteria (Burmaoglu et al., 2017).

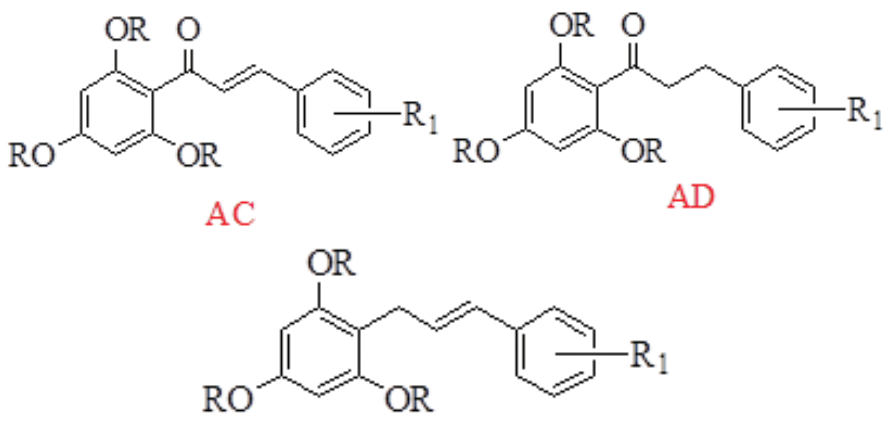

\begin{tabular}{cccccccc}
\hline Compound & $\begin{array}{c}\text { Main } \\
\text { structure }\end{array}$ & $\mathbf{R}$ & $\mathbf{R}_{\mathbf{1}}$ & Compound & $\begin{array}{c}\text { Main } \\
\text { structure }\end{array}$ & $\mathbf{R}$ & $\mathbf{R}_{\mathbf{1}}$ \\
\hline $\mathbf{1}$ & $\mathrm{AC}$ & $\mathrm{CH}_{3}$ & $\mathrm{H}$ & $\mathbf{8}$ & $\mathrm{AE}$ & $\mathrm{CH}_{3}$ & $3-\mathrm{F}$ \\
$\mathbf{2}$ & $\mathrm{AD}$ & $\mathrm{CH}_{3}$ & $\mathrm{H}$ & $\mathbf{9}$ & $\mathrm{AE}$ & $\mathrm{CH}_{3}$ & $2,5-\mathrm{di} \mathrm{F}$ \\
$\mathbf{3}$ & $\mathrm{AC}$ & $\mathrm{CH}_{3}$ & $2-\mathrm{F}$ & $\mathbf{1 0}$ & $\mathrm{AC}$ & $\mathrm{H}$ & $2-\mathrm{F}$ \\
$\mathbf{4}$ & $\mathrm{AC}$ & $\mathrm{CH}_{3}$ & $3-\mathrm{F}$ & $\mathbf{1 1}$ & $\mathrm{AC}$ & $\mathrm{H}$ & $2,5-\mathrm{di} \mathrm{F}$ \\
$\mathbf{5}$ & $\mathrm{AC}$ & $\mathrm{CH}_{3}$ & $4-\mathrm{F}$ & $\mathbf{1 2}$ & $\mathrm{AE}$ & $\mathrm{H}$ & $2-\mathrm{F}$ \\
$\mathbf{6}$ & $\mathrm{AC}$ & $\mathrm{CH}_{3}$ & $2,5-\mathrm{di} \mathrm{F}$ & & & & \\
$\mathbf{7}$ & $\mathrm{AE}$ & $\mathrm{CH}_{3}$ & $2-\mathrm{F}$ & $\mathbf{1 3}$ & $\mathrm{AE}$ & $\mathrm{H}$ & $2,5-\mathrm{di} \mathrm{F}$ \\
\hline
\end{tabular}

Recently, chalcones AF (1-5) have been reported by the coupling of 1-(2,6-dichloro-4-trifluoromethyl-phenyl)-pyrolidine2,5-dione, 1-(2,6-dichloro-4-trifluoromethyl-phenyl) piperidine2,6-dione, and various aromatic aldehydes in the acetic acid. The resultant products had shown antimicrobial properties (Rajput and Sayyed, 2017).<smiles></smiles>

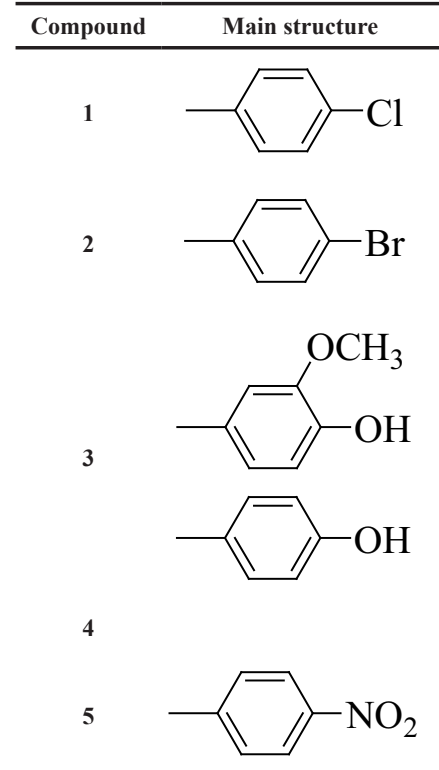

Chalcones AG (1-10) were prepared by carrying out Claisen-Schmidt's condensation between 2-acetyl-1methylpyrrole and 5-(aryl)-furfural analogs. The consequential products were tested to possess antimicrobial activities for five pathogenic bacteria and four fungi (Özdemir et al., 2017).<smiles>[R]c1ccc(/C=C/C(=O)C2CCCN2C)o1</smiles>

$$
\text { AG (1-10) }
$$

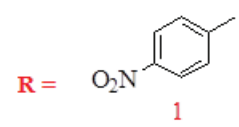<smiles>Cc1cccc([N+](=O)[O-])c1</smiles><smiles>Cc1ccccc1[N+](=O)[O-]</smiles><smiles>Cc1ccc(Cl)cc1[N+](=O)[O-]</smiles><smiles>Cc1ccccc1Cl</smiles><smiles>Cc1cccc(Cl)c1</smiles><smiles>Cc1ccc(Cl)cc1</smiles><smiles>Cc1cc(Cl)ccc1Cl</smiles><smiles>Cc1ccc(Cl)c(Cl)c1</smiles> 
A novel series of dehydroacetic acid chalcone-1,2,3triazole analogs AH (1-16) was designed and synthesized. The synthesized compounds were evaluated for antimicrobial activities against four bacterial and two fungal strains (Lal et al., 2018).

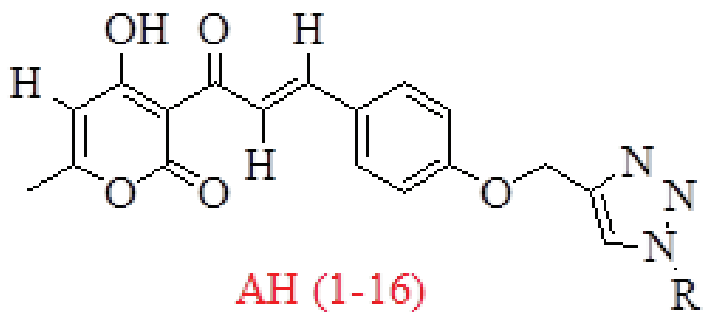

\begin{tabular}{cccc}
\hline Compound & \multicolumn{1}{c}{$\mathbf{R}$} & Compound & $\mathbf{R}$ \\
\hline $\mathbf{1}$ & $\mathrm{C}_{6} \mathrm{H}_{5}-$ & $\mathbf{9}$ & $3-\mathrm{BrC}_{6} \mathrm{H}_{4} \mathrm{CH}_{2}-$ \\
$\mathbf{2}$ & $2-\mathrm{CH}_{3} \mathrm{C}_{6} \mathrm{H}_{4} \mathrm{CH}_{2}^{-}$ & $\mathbf{1 0}$ & $4-\mathrm{BrC}_{6} \mathrm{H}_{4} \mathrm{CH}_{2}^{-}$ \\
$\mathbf{3}$ & $3-\mathrm{CH}_{3} \mathrm{C}_{6} \mathrm{H}_{4} \mathrm{CH}_{2}^{-}$ & $\mathbf{1 1}$ & $2-\mathrm{FC}_{6} \mathrm{H}_{4} \mathrm{CH}_{2}^{-}$ \\
$\mathbf{4}$ & $4-\mathrm{CH}_{3} \mathrm{C}_{6} \mathrm{H}_{4} \mathrm{CH}_{2}^{-}$ & $\mathbf{1 2}$ & $3-\mathrm{FC}_{6} \mathrm{H}_{4} \mathrm{CH}_{2}^{-}$ \\
$\mathbf{5}$ & $2-\mathrm{NO}_{2} \mathrm{C}_{6} \mathrm{H}_{4} \mathrm{CH}_{2}^{-}$ & $\mathbf{1 3}$ & $4-\mathrm{FC}_{6} \mathrm{H}_{4} \mathrm{CH}_{2}^{-}$ \\
$\mathbf{6}$ & $3-\mathrm{NO}_{2} \mathrm{C}_{6} \mathrm{H}_{4} \mathrm{CH}_{2}^{-}$ & $\mathbf{1 4}$ & $4-\mathrm{OCH}_{3} \mathrm{C}_{6} \mathrm{H}_{4}^{-}$ \\
7 & $4-\mathrm{NO}_{2} \mathrm{C}_{6} \mathrm{H}_{4} \mathrm{CH}_{2}^{-}$ & $\mathbf{1 5}$ & $4-\mathrm{BrC}_{6} \mathrm{H}_{4}^{-}$ \\
$\mathbf{8}$ & $2-\mathrm{BrC}_{6} \mathrm{H}_{4} \mathrm{CH}_{2-}^{-}$ & $\mathbf{1 6}$ & $4-\mathrm{NO}_{2} \mathrm{C}_{6} \mathrm{H}_{4}^{-}$ \\
\hline
\end{tabular}

\section{Chalcones as anticancer agents}

The synthesis of a novel sequence of $2^{\prime}, 5^{\prime}$-dialkoxyl chalcones BA (1-10) was carried out by condensing various aromatic ketones with various suitable and substituted benzaldehydes. The compounds were evaluated to show antitumor and chemopreventive activities (Cheng et al., 2008).<smiles>[R]Oc1ccc(O)c(C(=O)/C=C/c2ccc(C)s2)c1</smiles>

$\mathrm{BA}(1-10)$

\begin{tabular}{|c|c|c|c|c|c|}
\hline Compound & $\mathbf{R}$ & $R_{1}$ & Compound & $\mathbf{R}$ & $R_{1}$ \\
\hline 1 & $\mathrm{H}$ & $\mathrm{H}$ & 6 & $\mathrm{H}$ & \\
\hline 2 & $\mathrm{H}$ & $\mathrm{CH}_{2} \mathrm{CH}_{3}$ & 7 & $\mathrm{H}$ & \\
\hline 3 & $\mathrm{CH}_{2} \mathrm{CH}_{3}$ & $\mathrm{CH}_{2} \mathrm{CH}_{3}$ & 8 & $\mathrm{H}$ & $\mathrm{CH}_{3}$ \\
\hline 4 & $\mathrm{H}$ & & 9 & $\mathrm{CH}_{2} \mathrm{CH}_{3}$ & $\mathrm{CH}_{3}$ \\
\hline 5 & $\mathrm{H}$ & & 10 & & $\mathrm{CH}_{3}$ \\
\hline
\end{tabular}

A new quinazolinone-chalcone derivative was prepared by condensing the substituted aromatic aldehyde and substituted aromatic ketone in $\mathrm{Ba}(\mathrm{OH})$, and testing it for possessing anticancer activity (Wani et al., 2015).<smiles>COc1cc(/C=C/C(=O)c2cccc(-n3c(C)nc4ccccc4c3=O)c2)cc(OC)c1OC</smiles>

\section{Quinazolinone-Chalcone}

A novel series of chalcones BB (1-12) with 3-aryl thiophene-2-aryl and hetero aryl moieties was prepared and tested to show in vitro anticancer property for human colon cancer cell lines (Venkataramireddy et al., 2016).<smiles>[R]c1cccc(C(=O)/C=C/c2sccc2-c2ccccc2[R])c1</smiles>

$\mathrm{BB}(1-12)$

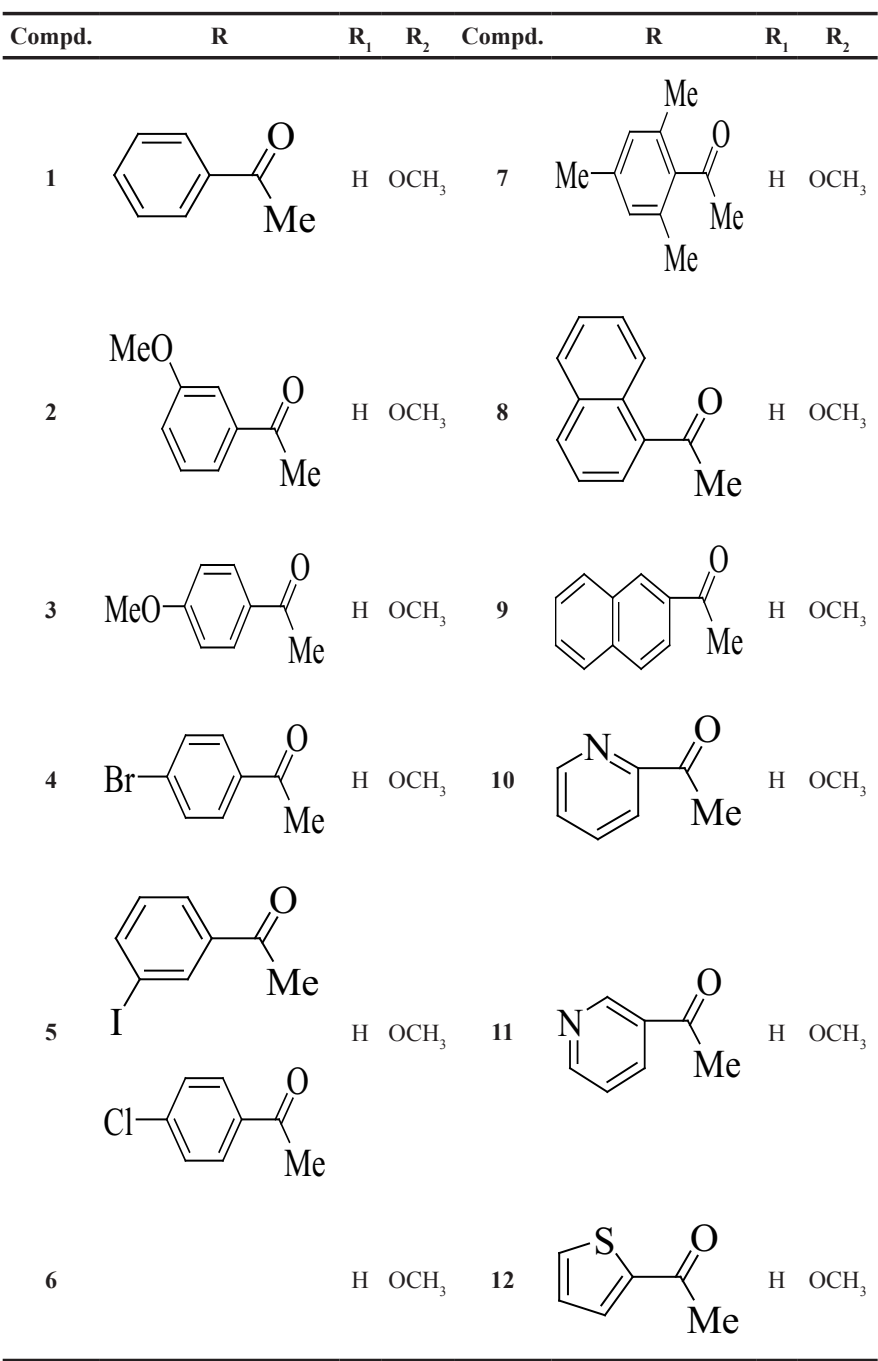


Ngameni et al. (2013) synthesized O-allyl chalcones BC (1-8) by condensing O-allyl vanillin with various acetophenones and reported antiproliferative activity of the synthesized chalcone derivative.<smiles>[R]c1ccc(C(=O)/C=C/c2ccc(OCC=C)c(OC)c2)c([R1])c1[R]</smiles>

\begin{tabular}{cccccccc}
\hline Compd. & $\mathbf{R}_{1}$ & $\mathbf{R}_{2}$ & $\mathbf{R}_{3}$ & Compd. & $\mathbf{R}_{1}$ & $\mathbf{R}_{2}$ & $\mathbf{R}_{3}$ \\
\hline $\mathbf{1}$ & $\mathrm{H}$ & $\mathrm{H}$ & $\mathrm{H}$ & $\mathbf{5}$ & $\mathrm{H}$ & $\mathrm{H}$ & $\mathrm{Me}$ \\
$\mathbf{2}$ & $\mathrm{OMe}$ & $\mathrm{H}$ & $\mathrm{H}$ & $\mathbf{6}$ & $\mathrm{H}$ & $\mathrm{Me}$ & $\mathrm{H}$ \\
$\mathbf{3}$ & $\mathrm{Me}$ & $\mathrm{H}$ & $\mathrm{Me}$ & $\mathbf{7}$ & $\mathrm{Me}$ & $\mathrm{H}$ & $\mathrm{H}$ \\
$\mathbf{4}$ & $\mathrm{H}$ & $\mathrm{OMe}$ & $\mathrm{H}$ & $\mathbf{8}$ & $\mathrm{H}$ & $\mathrm{H}$ & $\mathrm{OMe}$ \\
\hline
\end{tabular}

Novel derivatives of chalcones BD were prepared by condensing appropriate acetophenones and various benzaldehydes, followed by the reaction of the produced product (chalcone) with sodium acetate in ethanol to give flavanones $\mathrm{BE}$, which were evaluated as antiproliferative agents (Ketabforoosh et al., 2014).

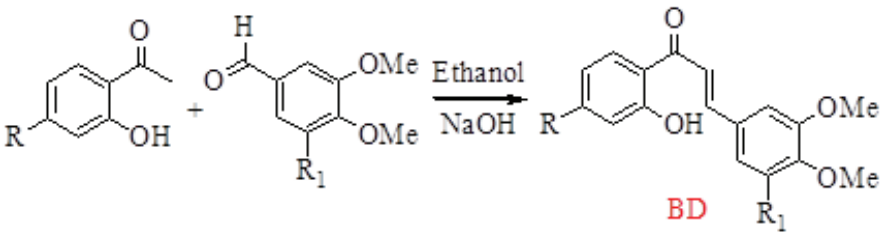<smiles>[R]c1ccc2c(=O)cc(-c3cc([R4])c(OC)c(OC)c3)oc2c1</smiles>

$\mathbf{R}=\mathrm{H} ; \mathbf{R}_{1}=\mathrm{H}, \mathrm{OMe}, \mathrm{Cl}, \mathrm{Br}$

\section{Chalcones as anti-inflammatory agents}

A novel chalcone 20-hydroxy-3,4-dichlorochalcone was synthesized which was evaluated for anti-inflammatory activity (Won et al., 2005).<smiles>O=C(/C=C/c1ccc(Cl)c(Cl)c1)c1ccccc1O</smiles>

\section{Dichol ochalcone}

Fluorinated chalcone analog was prepared by ClaisenSchmidt's condensation and by reacting with $\mathrm{SOCl}_{2} / \mathrm{EtOH}$, which possesses a powerful anti-inflammatory property (Hasan et al., 2012).<smiles>O=C(/C=C/c1ccc(F)cc1)c1ccc(O)cc1</smiles>

\section{Fluorinated chalcone}

Novel chalcones containing the isopropyl group (iPr), viz. compounds $\mathrm{CA}$ and $\mathrm{CB}$, were prepared by Claisen-Schmidt's condensation and were evaluated as active anti-inflammatory agents (Chen et al., 2013).<smiles>CCCOc1ccc(/C=C/C(=O)c2ccccc2OCCC)cc1</smiles>

A novel dihydroxy chloro chalcone was prepared and tested to be a potent anti-inflammatory agent (Zhang et al., 2010).<smiles>O=C(/C=C/c1ccc(Cl)cc1)c1ccc(O)cc1O</smiles>

\section{Dihydroxy chloro chalcone}

$2^{\prime}, 4$-dihydroxy-3', $4^{\prime}, 6^{\prime}$-trimethoxychalcone (CC) is a chalcone analog isolated from Chromolaena odorata (L.). It has been investigated for its anti-inflammatory activity against lipopolysaccharide-induced inflammation in RAW 264.7 macrophages. The results show that the compound significantly decreased the production of $\mathrm{NO}$ and pro-inflammatory cytokines, tumor necrosis factor- $\alpha$, interleukin-1 $\beta$, and IL-6. It also obstructed NF- $\mathrm{KB}$ activation by hindering the stimulation of inhibitor $\kappa \mathrm{B}$ kinase $\alpha / \beta$, degradation of inhibitor $\kappa \mathrm{B}$ (IкB) $\alpha$ and translocation of p65 NF- $\mathrm{kB}$ into the nucleus (Dhar et al., 2018).<smiles>COc1cc(OC)c2oc(-c3ccc(O)cc3)cc(=O)c2c1O</smiles>

In a recent study, a new series of chalcone analogs containing Apocynin and 5-Nitrofuran group have been reported. Compounds $\mathrm{CD}-\mathrm{CN}$ were prepared by Claisen-Schmidt's condensation, which was carried out under solvent-free conditions and was evaluated as active anti-inflammatory agents (Reddy and Kathale, 2018). 
<smiles>[R]C=CC(=O)c1ccc(OCc2ccc([N+](=O)[O-])o2)c(OC)c1</smiles>

$\mathrm{CD}-\mathrm{N}$<smiles>Cc1ccc(Br)cc1</smiles>

$\mathrm{R}=$<smiles>Cc1ccc(Cl)cc1</smiles>

CE<smiles>Cc1ccc(F)cc1</smiles>

$\mathrm{CF}$<smiles>Cc1ccc(OC(F)(F)F)cc1</smiles>

$\mathrm{CG}$<smiles>Cc1ccc([N+](=O)[O-])cc1</smiles>

$\mathrm{CH}$<smiles>COS(=O)(=O)c1ccc(C)cc1</smiles>

$\mathrm{CI}$<smiles>COc1ccc(C)cc1</smiles>

CJ<smiles>COc1ccc(C)c(OC)c1</smiles>

CK<smiles>COc1ccc(OC)c(C)c1</smiles>

$\mathrm{CL}$<smiles>COc1cccc(OC)c1C</smiles>

$\mathrm{CM}$<smiles>COc1cc(C)cc(OC)c1OC</smiles>

$\mathrm{CN}$

\section{Chalcones as antioxidants}

A sequence of novel derivatives of chalcones DA (1-6) with heterocyclic moiety was prepared by Claisen-Schmidt's condensation of 2-acetyl-5-chlorothiophene and various benzaldehyde derivatives with a catalytic amount of $\mathrm{NaOH}$ and methanol as solvents at room temperature and evaluated as antioxidant agents (Kumar et al., 2013).

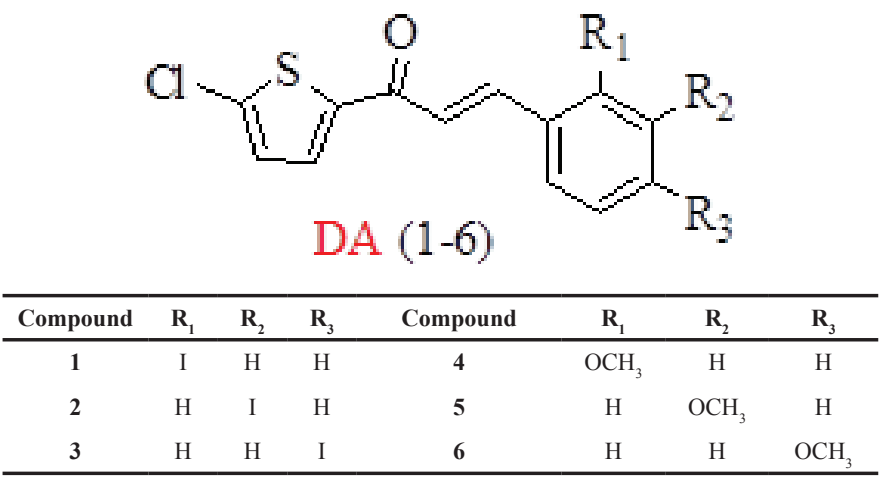

A new series of 2,4-dihydroxy chalcones DB (1-5) was prepared by the condensation of dihydroxy acetophenone and various benzaldehydes, followed by reaction with DMSO in the presence of iodine to give flavonoids DC (1-5), which were evaluated for antioxidant activity (Murti et al., 2013).<smiles>[R]c1cc(/C=C/C(=O)c2ccc(O)cc2O)c([R1])c([R])c1[R]</smiles>

DB (1-5)<smiles>[R]c1cc(-c2cc(=O)c3ccc(O)cc3o2)c([R4])c([Y1])c1[R]</smiles>

DC (1-5)

\begin{tabular}{ccccc}
\hline Compound & $\mathbf{R}_{1}$ & $\mathbf{R}_{2}$ & $\mathbf{R}_{3}$ & $\mathbf{R}_{4}$ \\
\hline $\mathbf{1}$ & $\mathrm{H}$ & $\mathrm{OH}$ & $\mathrm{H}$ & $\mathrm{H}$ \\
$\mathbf{2}$ & $\mathrm{H}$ & $\mathrm{OMe}$ & $\mathrm{H}$ & $\mathrm{H}$ \\
$\mathbf{3}$ & $\mathrm{H}$ & $\mathrm{Cl}$ & $\mathrm{H}$ & $\mathrm{H}$ \\
$\mathbf{4}$ & $\mathrm{H}$ & $\mathrm{H}$ & $\mathrm{NO}_{2}$ & $\mathrm{H}$ \\
$\mathbf{5}$ & $\mathrm{OMe}$ & $\mathrm{OH}$ & $\mathrm{H}$ & $\mathrm{OMe}$ \\
\hline
\end{tabular}

A new series of derivatives of chalcones was synthesized and tested for antioxidant activity (Wu et al., 2014).<smiles>[R2]C(=O)c1ccc(/C=C/C(=O)c2ccc(OC)cc2)cc1[R1]</smiles>

where $\mathbf{R}_{1}=\mathrm{H}, \mathrm{OCH}_{3} ; \mathbf{R}_{2}=\mathrm{CH}_{3}, \mathrm{C}_{2} \mathrm{H}_{5}, \mathrm{H}_{2} \mathrm{C}=\mathrm{CH}, \mathrm{Ph}$, 4-ClPh, 2-FPh, and Benzyl.

A novel chalcone, i.e., glycyglabrone, was isolated from the roots of liquorice (Glycyrrhiza glabra), along with three known derivatives, viz. licoagrochalcone, licochalcone, and kanzonol. The obtained chalcones DD, DE, and DF were found to possess antioxidant property (Chen et al., 2017).<smiles>[R]c1cc(C(=O)/C=C/c2ccc3c(c2OC)C=CC(C)(C)O3)ccc1O</smiles><smiles>COc1c(/C=C/C(=O)c2ccc(O)cc2)ccc(O)c1CC=C(C)C</smiles><smiles>CC(C)=CCc1cc(CC(O)C(=O)c2cc(CC=C(C)C)c(O)cc2O)ccc1O</smiles>

\section{Chalcones as an antiepileptic}

A new series of chalcones was prepared through ClaisenSchmidt's condensation, which was further tested for antiepileptic property (Sharma et al., 2013).<smiles>[R]c1ccc(/C=C/C(=O)c2ccc([R])cc2)cc1</smiles>

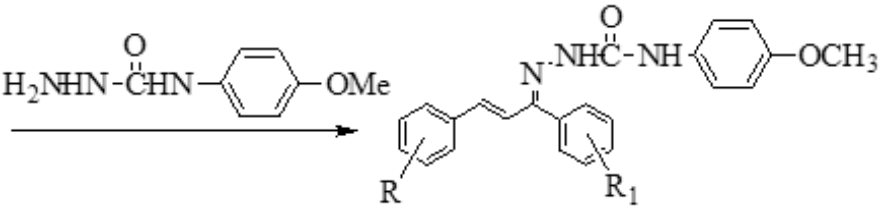


A new series of 3,5-diphenyl-2-pyrazoline-1carboxamide analogs 1-20 were prepared by the reaction of substituted chalcones with semicarbazide hydrochloride, which were evaluated to be potent antiepileptic derivatives (Siddiqui et al., 2010).<smiles>[R1]c1ccc(C(C)=O)cc1</smiles><smiles>[R1]c1ccc(C(=O)/C=C/c2ccc([R16])c([R2])c2)cc1</smiles><smiles>[R1]c1ccc(C2=NN(C(N)=O)C([Y])(c3ccc([R4])c([R4])c3)C2)cc1</smiles>

$1-20$

A new chalcone series EA (1-10) was synthesized which incorporated hydrazide derivatives and were evaluated as anticonvulsant agents (Kumar and Chauhan, 2015).

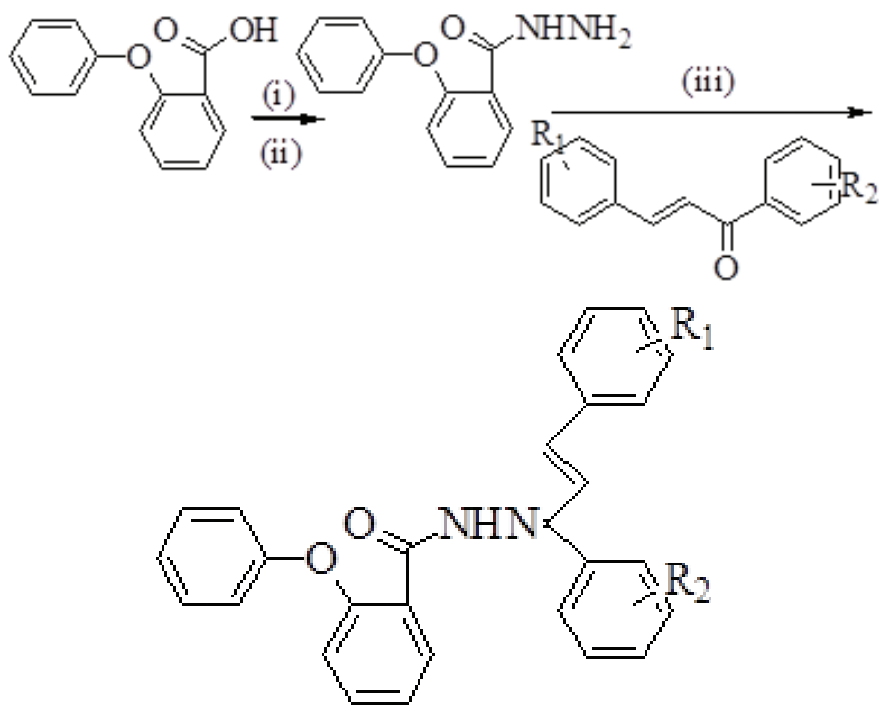

EA (i) $\mathrm{CH}_{3} \mathrm{OH}$, Conc. $\mathrm{H}_{2} \mathrm{SO}_{4}$, (ii) $\mathrm{NH}_{2} \mathrm{NH}_{2} \cdot \mathrm{H}_{2} \mathrm{O}$, and (iii) $\mathrm{CH}_{3} \mathrm{OH}$; Glacial acetic acid.

\begin{tabular}{cccccc}
\hline Compound & $\mathbf{R}_{1}$ & $\mathbf{R}_{2}$ & Compound & $\mathbf{R}_{1}$ & $\mathbf{R}_{2}$ \\
\hline $\mathbf{1}$ & $\mathrm{H}$ & $\mathrm{H}$ & $\mathbf{6}$ & $4-\mathrm{N}(\mathrm{Me})_{2}$ & $4-\mathrm{OH}$ \\
$\mathbf{2}$ & $3-\mathrm{NO}$ & $\mathrm{H}$ & $\mathbf{7}$ & $3-\mathrm{NO}_{2}$ & $4-\mathrm{Cl}$ \\
$\mathbf{3}$ & $4-\mathrm{Cl}$ & $\mathrm{H}$ & $\mathbf{8}$ & $4-\mathrm{N}(\mathrm{Me})_{2}$ & $4-\mathrm{F}$ \\
$\mathbf{4}$ & $4-\mathrm{Cl}$ & $4-\mathrm{OH}$ & $\mathbf{9}$ & $4-\mathrm{Cl}$ & $4-\mathrm{F}$ \\
$\mathbf{5}$ & $2-\mathrm{Cl}$ & $4-\mathrm{OH}$ & $\mathbf{1 0}$ & $4-\mathrm{NO}_{2}$ & $4-\mathrm{F}$ \\
\hline
\end{tabular}

\section{Chalcones as antidiabetic agents}

A novel series of chalcone-based aryl oxypropanolamine FA, FB, FC, and FD was synthesized and evaluated as powerful antidiabetic and antidyslipidemic agents (Shukla et al., 2017).<smiles>O=C(/C=C/c1ccc2c(c1)OCO2)c1ccc(Oc2ccccc2)cc1</smiles>

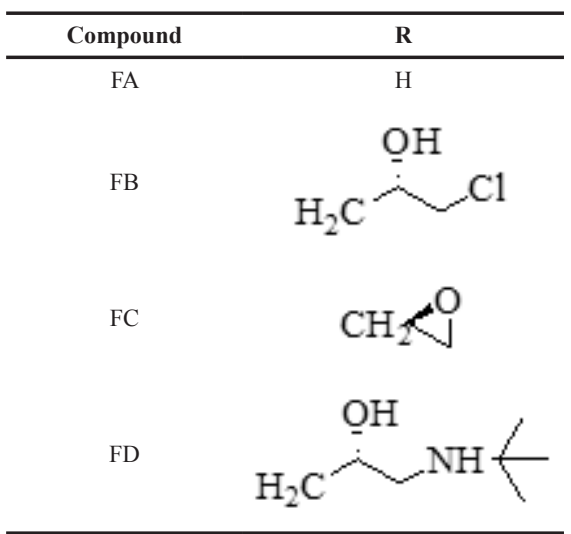

A new series of 60 derivatives of chalcone were synthesized having substitutions on Ring A or were unsubstituted by Claisen-Schmidt's condensation of some aromatic ketones with various benzaldehydes in $50 \% \mathrm{w} / \mathrm{\textrm {KOH }} / \mathrm{H}_{2} \mathrm{O}$ with solvent ethanol. Out of these 60 derivatives, 12 derivatives were found to be active as antidiabetic agents (Hsieh et al., 2012).

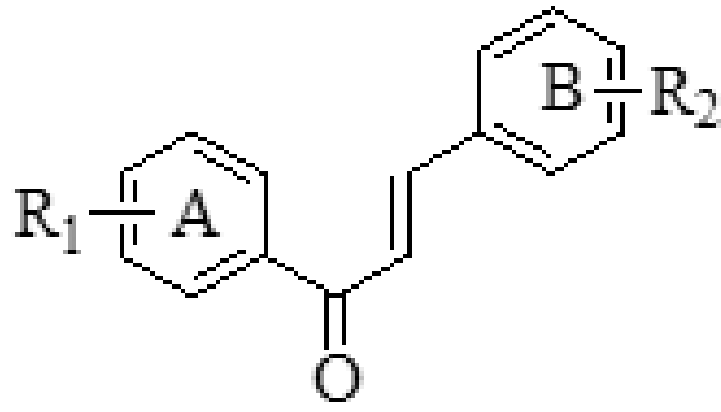
$-\mathrm{OCH}_{2} \mathrm{O}-$

$\mathbf{R}_{1}=\mathrm{H}, \mathrm{OH}, \mathrm{F}, \mathrm{Cl}, \mathrm{Br}, \mathrm{I} ; \quad \mathbf{R}_{\mathbf{2}}=\mathrm{H}, \mathrm{OMe}, \mathrm{OBn}$,

A series of chalcone FE and its 2-pyrazoline analogs FF, FG, FH, FI, and FJ was prepared and tested to be antidiabetic agents (Emayavaramban et al., 2013). 
<smiles>Cc1ccc(/C=C/C(=O)c2ccc(C)cc2)cc1</smiles><smiles>Cc1ccc(C2=NN(C(N)=O)C(c3ccc(C)cc3)C2)cc1</smiles>

FF<smiles>CCCSN1N=C(c2ccc(C)cc2)CC1c1ccc(C)cc1</smiles><smiles>Cc1ccc(C2=NN(C(=O)c3ccccc3)C(c3ccc(C)cc3)C2)cc1</smiles>
$\mathrm{FH}$<smiles>Cc1ccc(C2=NN(c3ccccc3)C(c3ccc(C)cc3)C2)cc1</smiles>

Chalcones, which are derivatives of isoliquiritigenin and liquiritigenin and have flavonoid moiety, viz. FK, FL, FM, FN, FO, FP, FQ, FR, FS, FT, and FU, were synthesized and evaluated for their antidiabetic activity (Gaur et al., 2014).<smiles>[R]c1ccc(/C=C/C(=O)c2ccc([R4])cc2[R1])cc1</smiles>

\begin{tabular}{cccc}
\hline Compound & $\mathbf{R}_{1}$ & $\mathbf{R}_{2}$ & $\mathbf{R}_{3}$ \\
\hline $\mathrm{FK}$ & $\mathrm{OH}$ & $\mathrm{OH}$ & $\mathrm{OH}$ \\
$\mathrm{FM}$ & $\mathrm{OH}$ & $\mathrm{OOCCH}_{3}$ & $\mathrm{OOCCH}_{3}$ \\
$\mathrm{FN}$ & $\mathrm{OCH}_{3}$ & $\mathrm{OCH}_{3}$ & $\mathrm{OH}$ \\
$\mathrm{FO}$ & $\mathrm{OCH}_{3}$ & $\mathrm{OCH}_{3}$ & $\mathrm{OOCCH}_{3}$ \\
$\mathrm{FP}$ & $\mathrm{OCH}_{3}$ & $\mathrm{OCH}_{3}$ & $\mathrm{H}$ \\
$\mathrm{FS}$ & $\mathrm{OCH}_{3}$ & $\mathrm{OCH}_{3}$ & $\mathrm{OCOC}_{6} \mathrm{H}_{5}$ \\
\hline
\end{tabular}<smiles>[R]C1=CC(c2ccc([R3])cc2)Oc2cc([R1])ccc21</smiles>

\begin{tabular}{cccc}
\hline Compound & $\mathbf{R}_{1}$ & $\mathbf{R}_{\mathbf{2}}$ & $\mathbf{R}_{\mathbf{3}}$ \\
\hline $\mathrm{FL}$ & $\mathrm{OH}$ & $\mathrm{O}$ & $\mathrm{OH}$ \\
$\mathrm{FQ}$ & $\mathrm{OOCCH}_{3}$ & $\mathrm{O}$ & $\mathrm{OOCCH}_{3}$ \\
FR & $\mathrm{OH}$ & $\mathrm{O}$ & $\mathrm{OOCCH}_{3}$ \\
FT & $\mathrm{OCOC}_{6} \mathrm{H}_{5}$ & $\mathrm{O}$ & $\mathrm{OCOC}_{6} \mathrm{H}_{5}$ \\
FU & $\mathrm{OH}$ & $\mathrm{NOH}$ & $\mathrm{OH}$ \\
\hline
\end{tabular}

A novel series of chalconeimines, FV, FW, FX, FY, and FZ, was prepared and evaluated for antidiabetic activity via invitro $\alpha$-amylase inhibition activity (Balu et al., 2019).<smiles>Oc1cc(/C=C\C(=Nc2cccnc2Cl)c2ccc3c(c2)OCO3)ccc1OC(F)C(F)F</smiles>

FV<smiles>Oc1cc(/C=C\C(=Nc2ccc(Cl)cn2)c2ccc3c(c2)OCO3)ccc1OC(F)F</smiles>

FX<smiles>Cc1ccncc1/N=C(\C=C/c1ccc(OC(F)F)c(O)c1)c1ccc2c(c1)OCO2</smiles>

FY<smiles>[R2]C(F)Oc1ccc(/C=C\C(=Nc2ccc(C)cn2)c2ccc3c(c2)OCO3)cc1O</smiles>

\section{Chalcones as antihypertensive agents}

Novel chalcones GA (1-7) were synthesized, consisting of pyrimidine as the basic moiety, and tested to be potent antihypertensive agents (Bukhari et al., 2013).<smiles>[R]C1C=C(CN=[N+]c2ccc(C(=O)O)cc2)N=C(O)N1</smiles><smiles>[14CH3]</smiles>

\begin{tabular}{cccc}
\hline Compound & $\mathbf{R}$ & Compound & $\mathbf{R}$ \\
\hline $\mathbf{1}$ & $\mathrm{C}_{6} \mathrm{H}_{5}$ & $\mathbf{5}$ & $4-\mathrm{OCH}_{3} \mathrm{C}_{6} \mathrm{H}_{5}$ \\
$\mathbf{2}$ & $2-\mathrm{OH} \mathrm{C}_{6} \mathrm{H}_{5}$ & $\mathbf{6}$ & $3-\mathrm{NO}_{2} \mathrm{C}_{6} \mathrm{H}_{5}$ \\
$\mathbf{3}$ & $4-\mathrm{OH} \mathrm{C}_{6} \mathrm{H}_{5}$ & 7 & $2-\mathrm{Cl} \mathrm{C}_{6} \mathrm{H}_{5}$ \\
4 & $2-\mathrm{NO}_{2} \mathrm{C}_{6} \mathrm{H}_{5}$ & & \\
\hline
\end{tabular}


Studies were carried out on vasorelaxant and antihypertensive properties of dihydrospinochalcone and Isocordoin isolated from Lonchocarpus xuul (Avila-Villarreal et al., 2013).<smiles>CC(C)=CCc1cc(C(=O)CCc2ccccc2)c(O)c(CC=C(C)C)c1O</smiles>

Dihydrospinochalcone

Isocordin

Novel chalcones with quinoline in their structure GB were prepared and tested to have powerful antihypertensive moiety (Kumar et al., 2015).<smiles>Cc1nc2c(Br)cc(Br)cc2cc1C(=O)/C=C/C12C=CC=CC1C=c1ccccc1=C2</smiles>

$\mathrm{GB}$

\section{Chalcones as antimalarial agents}

Three aminoalkylated derivatives of chalcones HA (13) were synthesized by Claisen-Schmidt's condensation between chloroacetophenone and vanillin. After this reaction, addition of the amine group was carried out through Mannich's reaction. The synthesized derivatives were evaluated for antimalarial activity against Plasmodium falciparum strain (3D7) and molecular docking was also performed. Molecular docking and biological evaluation shows that compound HA2 was the most active derivative among the synthesized derivatives (Syahri et al., 2020).<smiles>[R]Cc1cc(/C=C/C(=O)c2ccccc2)cc(OC)c1O</smiles>

\begin{tabular}{cc}
\hline Compound & $\boldsymbol{R}$ \\
\hline $\mathbf{1}$ & Morpholine \\
$\mathbf{2}$ & Piperidine \\
$\mathbf{3}$ & Diethylamine \\
\hline
\end{tabular}

Table 1. Physical properties of chalcone.

\begin{tabular}{lc}
\hline IUPAC name & trans-1,3-diaryl-2- propen-1-one \\
\hline Molecular formula & $\mathrm{C}_{15} \mathrm{H}_{12} \mathrm{O}$ \\
Molar mass & $208.26 \mathrm{~g} \mathrm{~mol}^{-1}$ \\
Exact mass & 208.088815 \\
Density & $1.071 \mathrm{~g} / \mathrm{mol}^{3}$ \\
Melting point & $55^{\circ} \mathrm{C}-57^{\circ} \mathrm{C}$ \\
Boiling point & $345^{\circ} \mathrm{C}-348^{\circ} \mathrm{C}$ \\
\hline
\end{tabular}

\section{CONCLUSION}

From this review, it can be stated that chalcones and their derivatives show a wide spectrum of biological activities, viz anticancer, antimicrobial, anticonvulsant, antioxidant, antiinflammatory activities, etc. That is why the attention of scientists has increased towards chalcones in searching for novel and biologically potent derivatives from them.

\section{CONFLICT OF INTEREST}

None.

\section{FUNDING}

None.

\section{REFERENCES}

Abe I, Sano Y, Takahashi Y, Noguchi H. Site-directed mutagenesis of benzalacetone synthase the role of PHE215 in plant type III polyketide synthases. J Biol Chem, 2003; 278(27):25218-26.

Adole VA, Jagdale BS, Pawar TB, Sagane AA. Ultrasound promoted stereoselective synthesis of 2, 3-dihydrobenzofuran appended chalcones at ambient temperature. S Afr J Chem, 2020; 73:35-43.

Avila-Villarreal G, Hernández-Abreu O, Hidalgo-Figueroa S, Navarrete-Vázquez G, Escalante-Erosa F, Peña-Rodríguez LM, VillalobosMolina R, Estrada-Soto S. Antihypertensive and vasorelaxant effects of dihydrospinochalcone-A isolated from lonchocarpus xuul lundell by NO production: computational and ex vivo approaches. Phytomedicine, 2013; 20(14):1241-6.

Awasthi SK, Mishra N, Kumar B, Sharma M, Bhattacharya A, Mishra LC, Bhasin VK. Potent antimalarial activity of newly synthesized substituted chalcone analogs in vitro. Med Chem Res, 2009; 18(6):407-20.

Balu P, Jas JS, Govindaraj M. Design and evaluation of chalconeimine derivatives as $\alpha$-amylase inhibitors. Bioinformation, 2019; 15(7):523-9.

Bandgar BP, Gawande SS, Bodade RG, Totre JV, Khobragade $\mathrm{CN}$. Synthesis and biological evaluation of simple methoxylated chalcones as anticancer, anti-inflammatory and antioxidant agents. Bioorg Med Chem, 2010; 18(3):1364-70.

Baviskar BA, Baviskar BB, Shiradkar MR, Deokate UA, Khadabadi SS. Synthesis and antimicrobial activity of some vovel benzimidazolyl chalcones. Eur J Chem, 2009; 6(1):196-200.

Beltramino R, Penenory A, Buceta AM. An open-label, randomised multicentre study comparing the efficacy and safety of CYCLO 3 FORT versus hydroxylethylrutoside in chronic venous lymphatic insufficiency. Int Angiol, 1999; 18(4):337-42.

Beltramino R, Penenory A, Buceta AM. An open-label, randomized multicenter study comparing the efficacy and safety of Cyclo 3 Fort ${ }^{\circ}$ versus hydroxyethylrutoside in chronic venous lymphatic insufficiency. Angiol, 2000; 51(7):535-44.

Benouda H, Bouchal B, Challioui A, Oulmidi A, Harit T, Malek F, Riahi A, Bellaoui M, Bouammali B. Synthesis of a series of chalcones and related flavones and evaluation of their antibacterial and antifungal activities. Lett Drug Des Discov, 2019; 16(1):93-100.

Bianco A, Cavarischia C, Farina A, Guiso M, Marra C. A new synthesis of flavonoids via Heck reaction. Tetrahedron Lett, 2003; 44(51):9107-9.

Blass $\mathrm{BE} . \mathrm{KF} / \mathrm{Al}_{2} \mathrm{O}_{3}$ mediated organic synthesis. Tetrahedron, 2002; 46(58):9301-20.

Braun RU, Ansorge M, Mueller TJ. Coupling-isomerization synthesis of chalcones. Chemistry, 2006; 12(35):9081-94.

Bukhari SN, Butt AM, Amjad MW, Ahmad W, Shah VH, Trivedi AR. Synthesis and evaluation of chalcone analogues based pyrimidines as angiotensin converting enzyme inhibitors. Pak J Biol Sci, 2013; 16(21):1368-72. 
Burmaoglu S, Algul O, Gobek A, Aktas Anil D, Ulger M, Erturk BG, Kaplan E, Dogen A, Aslan G. Design of potent fluoro-substituted chalcones as antimicrobial agents. J Enzyme Inhib Med Chem, 2017; 32(1):490-5.

Calvino V, Picallo M, López-Peinado AJ, Martín-Aranda RM, Durán-Valle CJ. Ultrasound accelerated Claisen-Schmidt condensation: a green route to chalcones. Appl Surf Sci, 2006; 252(17):6071-4.

Cancio N, Costantino AR, Silbestri GF, Pereyra MT. Ultrasoundassisted syntheses of chalcones: experimental design and optimization. In Multidisciplinary Digital Publishing Institute Proceedings, Bahía Blanca, Argentina, 2019, 41(1), p 13

Chen JJ, Cheng MJ, Shu CW, Sung PJ, Lim YP, Cheng LY, Wang SL, Chen LC. A new chalcone and antioxidant constituents of Glycyrrhiza glabra. Chem Nat Compd, 2017; 53(4):632-4.

Chen YH, Wang WH, Wang YH, Lin ZY, Wen CC, Chern CY. Evaluation of the anti-inflammatory effect of chalcone \& chalcone analogues in a zebrafish model. Molecules, 2013; (18):2052-60.

Cheng JH, Hung CF, Yang SC, Wang JP, Won SJ, Lin CN. Synthesis and cytotoxic, anti-inflammatory, and anti-oxidant activities of 2', 5'-dialkoxylchalcones as cancer chemopreventive agents. Bioorg Med Chem, 2008; 16(15):7270-6.

Cheng MS, Li R, Kenyon G. A solid phase synthesis of chalcones by claisen-schmidt condensations. Chin Chem Lett, 2000; 11(10):851-4.

Choudhary AN, Kumar A, Juygal V. Design, Synthesis and evaluation of chalcone derivatives as anti-inflammatory, antioxidant and antiulcer agents. Lett Drug Des Discov, 2012; 9(5):479-88.

Dhar R, Kimseng R, Chokchaisiri R, Hiransai P, Utaipan T, Suksamrarn A, Chunglok W. 2', 4-Dihydroxy-3', 4', 6'-trimethoxychalcone from chromolaena odorata possesses anti-inflammatory effects via inhibition of NF- $\mathrm{KB}$ and p38 MAPK in lipopolysaccharide-activated RAW 264.7 macrophages. Immunopharmacol Immunotoxicol, 2018; 40(1):4351

Echeverria C, Santibañez JF, Donoso-Tauda O, Escobar C, Ramirez-Tagle R. Structural antitumoral activity relationships of synthetic chalcones. Int J Mol Sci, 2009; 10(1):221-31.

Emayavaramban M, Santhi N, Gopi C, Manivannan C, Raguraman A. Synthesis, characterization and anti-diabetic activity of 1,3 , 5-triaryl-2-pyrazolines in acetic acid solution under ultrasound irradiation. Int Lett Chem Phys Astron, 2013; 9:172-85.

Fringuelli F, Pizzo F, Vittoriani C, Vaccaro L. Polystyrylsupported TBD as an efficient and reusable catalyst under solvent-free conditions. Chem Commun, 2004; (23):2756-7.

$\mathrm{Fu} \mathrm{ZY}$, Jin QH, Qu YL, Guan LP. Chalcone derivatives bearing chromen or benzo [f] chromen moieties: design, synthesis and evaluations of anti-inflammatory, analgesic, selective COX-2 inhibitory activities. Bioorg Med Chem Lett, 2019; 29:1909-12.

Gall EL, Texier-Boullet F, Hamelin J. Simple access to $\alpha$, $\beta$ unsaturated ketones by acid-catalyzed solvent-free reactions. Synth Commun, 1999; 29(20):3651-7.

Gan FF, Zhang R, Ng HL, Karuppasamy M, Seah W, Yeap WH, Ong SM, Hadadi E, Wong SC, Chui WK, Chew EH. Novel dual-targeting anti-proliferative dihydrotriazine-chalcone derivatives display suppression of cancer cell invasion and inflammation by inhibiting the NF- $\mathrm{KB}$ signaling pathway. Food Chem Toxicol, 2018; 116:238-48.

Gaonkar SL, Vignesh UN. Synthesis and pharmacological properties of chalcones: a review. Res Chem Intermediat, 2017; 43(11):6043-77.

Gaur R, Yadav KS, Verma RK, Yadav NP, Bhakuni RS. In vivo anti-diabetic activity of derivatives of isoliquiritigenin and liquiritigenin. Phytomedicine, 2014; 21(4):415-22.

Gerhauser C. Cancer chemopreventive potential of apples, apple juice and apple components. Planta Med, 2008; 74(13):1608-24.

Hasan SA, Elias AN, Jwaied AH, Khuodaer AR, Hussain SA. Synthesis of new fluorinated chalcone derivative with anti-inflammatory Activity. Int J Pharm Pharm Sci, 2012; 4(5):430-4
Higuchi K, Watanabe T, Tanigawa T, Tominaga K, Fujiwara Y, Arakawa T. Sofalcone, a gastroprotective drug, promotes gastric ulcer healing following eradication therapy for Helicobacter pylori: a randomized controlled comparative trial with cimetidine, an $\mathrm{H}_{2}$-receptor antagonist. J Gastroenterol Hepatol, 2010; 25:S155-60.

Hofmann E, Webster J, Do T, Kline R, Snider L, Hauser Q, Higginbottom G, Campbell A, Ma L, Paula S. Hydroxylated chalcones with dual properties: xanthine oxidase inhibitors and radical scavengers. Bioorg Med Chem, 2016; 24(4):578-87.

Hsieh CT, Hsieh TJ, El-Shazly M, Chuang DW, Tsai YH, Yen CT, Wu SF, Wu YC, Chang FR. Synthesis of chalcone derivatives as potential anti-diabetic agents. Bioorg Med Chem Lett, 2012; 22(12):3912-5.

Hsieh CY, Ko PW, Chang YJ, Kapoor M, Liang YC, Lin HH, Horng JC, Hsu MH. Design and synthesis of benzimidazole-chalcone derivatives as potential anticancer agents. Molecules, 2019; 24(18):3259.

Hsieh CT, Ötvös SB, Wu YC, Mándity IM, Chang FR, Fülöp F. Highly selective continuous flow synthesis of potentially bioactive deuterated chalcone derivatives. Chempluschem, 2015; 80(5):859-64.

Insuasty B, Ramírez J, Becerra D, Echeverry C, Quiroga J, Abonia R, Robledo SM, Vélez ID, Upegui Y, Munoz JA, Ospina V. An efficient synthesis of new caffeine-based chalcones, pyrazolines and pyrazolo $[3,4-b][1,4]$ diazepines as potential antimalarial, antitrypanosomal and antileishmanial agents. Euro J Med Chem, 2015; 93:401-13.

Kakati D, Sarma JC. Microwave assisted solvent free synthesis of 1, 3-diphenylpropenones. Chem Cent J, 2011; 5(1):8-12.

Kaur H, Narasimhan B. Synthesis, characterization, antimicrobial and antioxidant potential of diazenyl chalcones. Curr Top Med Chem, 2018; 18(10):844-56

Ketabforoosh SH, Kheirollahi A, Safavi M, Esmati N, Ardestani SK, Emami S, Firoozpour L, Shafiee A, Foroumadi A. Synthesis and anticancer activity evaluation of new dimethoxylated chalcone \& flavanone analogs. Arch Pharm (Weinheim), 2014; 347(11):853-60.

Khanapure S, Jagadale M, Bansode P, Choudhari P, Rashinkar G. Anticancer activity of ruthenocenyl chalcones and their molecular docking studies. J Mol Struct, 2018; 1173:142-7.

Kolot C, Rodriguez-Mateos A, Feliciano R, Bottermann K, Stahl W. Bioavailability of naringenin chalcone in humans after ingestion of cherry tomatoes. Int J Vitam Nutr Res, 2019; 90(5-6):411-6.

Kostanecki SV, Tambor J. Synthesis, characterization and biological evaluation of some novel chalcone derivatives containing imidazo[1,2-a]pyridine moiety. J Chem Ber, 1899; 32:1921-9.

Kumar C, Loh WS, Ooi C, Quah C, Fun HK. Structural correlation of some heterocyclic chalcone analogues and evaluation of their antioxidant potential. Molecules, 2013; 18(10):11996-2011.

Kumar H, Devaraji V, Joshi R, Jadhao M, Ahirkar P, Prasath R, Bhavana P, Ghosh SK. Antihypertensive activity of a quinoline appended chalcone derivative and its site specific binding interaction with a relevant target carrier protein. RSC Adv, 2015; 5(80):65496-513.

Kumar N, Chauhan LS. Synthesis and anticonvulsant activity of some flavones incorporated hydrazide derivatives. Int J Pharm Clin Res, 2015; 7(4):317-22.

Lal K, Yadav P, Kumar A, Kumar A, Paul AK. Design, synthesis, characterization, antimicrobial evaluation and molecular modeling studies of some dehydroacetic acid-chalcone-1, 2, 3-triazole hybrids. Bioorg Chem, 2018; 77:236-44

Li J, Li D, Xu Y, Guo Z, Liu X, Yang H, Wu L, Wang L. Design, synthesis, biological evaluation, and molecular docking of chalcone derivatives as anti-inflammatory agents. Bioorg Med Chem Lett, 2017; 27(3):602-6.

Li JT, Yang WZ, Wang SX, Li SH, Li TS. Improved synthesis of chalcones under ultrasound irradiation. Ultrason Sonochem, 2002; 9(5):237-9.

Liu M, Wilairat P, Go ML. Antimalarial alkoxylated and hydroxylated chalones: structure-activity relationship analysis. $\mathrm{J} \mathrm{Med}$ Chem, 2001; 44(25):4443-52. 
Liu Y, Lund JA, Murch SJ, Brown PN. Single-lab validation for determination of kavalactones and flavokavains in piper methysticum (Kava). Planta Med, 2018; 84(16):1213-8.

Mahapatra DK, Bharti SK, Asati V. Chalcone derivatives: antiinflammatory potential and molecular targets perspectives. Curr Top Med Chem, 2017; 17(28):3146-69.

Mahapatra DK, Bharti SK, Asati V. Chalcone scaffolds as antiinfective agents: structural and molecular target perspectives. Eur J Med Chem, 2015; 101:496-524.

Mariadoss AV, Vinyagam R, Rajamanickam V, Sankaran V, Venkatesan S, David E. Pharmacological aspects and potential use of phloretin: a systemic review. Mini Rev Med Chem, 2019; 19(13):1060-7.

Md Idris MH, Amin SNM, Selvaraj M, Jamari H, Kek TL. Highthroughput structure-based drug design of Chalcones Scaffolds as dual inhibitor of cyclooxygenase-2 and microsomal prostaglandin E synthase-1. J Pharm Sci Emerg Drugs, 2018; 6(1):1-14.

Min J, Li X, HuANG KE, Tang H, Ding X, Qi C, Qin X, Xu ZH. Phloretin induces apoptosis of non-small cell lung carcinoma A549 cells via JNK1/2 and p38 MAPK pathways. Oncol Rep, 2015; 34(6):2871-9.

Mistry RN, Desai KR. Microwave studies on synthesis of some new heterocyclic chalcone and pyrimidine-2-thione derivatives and their antibacterial activity. Asian J Chem, 2004; 16(1):201.

Monga V, Goyal K, Steindel M, Malhotra M, Rajani DP, Rajani SD. Synthesis and evaluation of new chalcones, derived pyrazoline and cyclohexenone derivatives as potent antimicrobial, antitubercular and antileishmanial agents. Med Chem Res, 2014; 23(4):2019-32.

Murti Y, Goswam A, Mishra P. Synthesis and antioxidant activity of some chalcones and flavanoids. Inter J Pharm Tech Res, 2013; 5:811-8.

Ngameni B, Kuete V, Ambassa P, Justin K, Marlyse ML, Tchoukoua A, Roy R, Ngadjui BT, Tetsuya M. Synthesis and evaluation of anticancer activity of O-allyl chalcone derivatives. Med Chem, 2013; 3(3):233-7.

Ötvös S, Hsieh CT, Wu YC, Li JH, Chang FR, Fülöp F. Continuous-flow synthesis of deuterium-labelled antidiabetic chalcones: Studies towards the selective deuteration of the alkynone core. Molecules, 2016; 21(3):318-28.

Özdemir A, Altıntop MD, Sever B, Gençer HK, Kapkaç HA, Atlı Ö, Baysal M. A new series of pyrrole-based chalcones: synthesis and evaluation of antimicrobial activity, cytotoxicity and genotoxicity. Molecules, 2017; 22(12):2112.

Padaratz P, Fracasso M, De Campos-Buzzi F, Corrêa R, Niero R, Monache FD, Cechinel-Filho V. Antinociceptive activity of a new benzofuranone derived from a chalcone. Basic Clin Pharmacol Toxicol, 2009; 105(4):257-61.

Pingaew R, Saekee A, Mandi P, Nantasenamat C, Prachayasittikul S, Ruchirawat S, Prachayasittikul V. Synthesis, biological evaluation and molecular docking of novel chalcone-coumarin hybrids as anticancer and antimalarial agents. Euro J Med Chem, 2014; 85:65-76.

Pinner KD, Wales CT, Gristock RA, Vo HT, So N, Jacobs AT. Flavokawains A and B from kava (Piper methysticum) activate heat shock and antioxidant responses and protect against hydrogen peroxide-induced cell death in HepG2 hepatocytes. Pharm Biol, 2016; 54(9):1503-12.

Polo E, Ibarra-Arellano N, Prent-Peñaloza L, Morales-Bayuelo A, Henao J, Galdámez A, Gutiérrez M. Ultrasound-assisted synthesis of novel chalcone, heterochalcone and bis-chalcone derivatives and the evaluation of their antioxidant properties and as acetylcholinesterase inhibitors. Bioorg Chem. 2019; 90:103034.

Prasad YR, Kumar PP, Kumar PR, Rao AS. Synthesis and antimicrobial activity of some new chalcones of 2-acetyl pyridine. J Chem, 2008; 5(1):144-8.

Rahman A, Qureshi R, Kiran M, Ansari FL. Electron affinities, solvation energies and redox potentials of some chalcones: substituents effect and correlation with semi-empirical MO energies. Turk J Chem, 2007; 31(1): 25-34.

Rajput SS, Sayyed RA. Synthesis and evaluation of antimicrobial activity of some novel chalcones of 2,6-dichloro-4-trifluoro methyl aniline. Heterocycl Lett, 2017; 7(2):333-9.
Rammohan A, Reddy JS, Sravya G, Rao CN, Zyryanov GV. Chalcone synthesis, properties and medicinal applications: a review. Environ Chem Lett, 2020; 18:433-58.

Rathore MM, Rajput PR, Parhate VV. Synthesis and antimicrobial activity of some chalcones and flavones. Int J Chem Phys Sci, 2015; (4):473-7.

Reddy AK, Kathale NE. Synthesis, Characterization and antiinflammatory activity of chalcone derivatives linked with Apocynin and 5-nitrofuran moiety. Asian J Chem, 2018; 30(2):312-6.

Reiland H, Slavin J. Systematic review of pears and health. Nutr Today, 2015; 50(6):301-5.

Rossi GV, Avellino JD. An evaluation of the antihistaminic activity of a new series of chalcone derivatives. Am J Pharm Sci Support Public Health, 1957; 129(9):324-31.

Rueping M, Bootwicha T, Baars H, Sugiono E. Continuous-flow hydration-condensation reaction: Synthesis of $\alpha, \beta$-unsaturated ketones from alkynes and aldehydes by using a heterogeneous solid acid catalyst. Beilstein J Org Chem, 2011; 7(1):1680-7.

Sahu NK, Balbhadra SS, Choudhary J, Kohli DV. Exploring pharmacological significance of chalcone scaffold: a review. Curr Med Chem, 2012; 19(2):209-25.

Sasaki C, Ichitani M, Kunimoto KK, Asada C, Nakamura Y. Extraction of arbutin and its comparative content in branches, leaves, stems and fruits of Japanese pear Pyrus pyrifolia cv. Kousui. Biosci Biotechnol Biochem, 2014; 78(5):874-7.

Sashidhara KV, Kumar A, Kumar M, Sarkar J, Sinha S. Synthesis and in vitro evaluation of novel coumarin-chalcone hybrids as potential anticancer agents. Bioorg Med Chem Lett, 2010; 20(24):7205-11.

Sayed M, Kamal El-Dean AM, Ahmed M, Hassanien R. Synthesis, characterization, and screening for anti-inflammatory and antimicrobial activity of novel indolyl chalcone derivatives. J Heterocycl Chem, 2018; 55(5):1166-75.

Selepe M, Van Heerden F. Application of the Suzuki-Miyaura reaction in the synthesis of flavonoids. Molecules, 2013; 18(4):4739-65.

Sharma CS, Shekhawat KS, Chauhan CS, Kumar N. Synthesis and anticonvulsant activity of some chalcone derivatives. J Chem Pharm Res, 2013; 5(10):450-4.

Shukla P, Satyanarayana M, Verma PC, Tiwari J, Dwivedi AP, Srivastava R, Rehuja N, Srivastava SP, Gautam S, Tamrakar AK, Dwivedi AK. Chalcone-based aryloxypropanolamine as a potential antidiabetic and antidyslipidaemic agent. Curr Sci, 2017; 112:1675-89.

Siddiqui AA, Rahman MA, Shaharyar M, Mishra R. Synthesis and anticonvulsant activity of some substituted 3, 5-diphenyl-2-pyrazoline1-carboxamide derivatives. Chem Sci J, 2010; 8:1-10.

Slimestad R, Verheul M. Properties of chalconaringenin and rutin isolated from cherry tomatoes. J Agric Food Chem, 2011; 59(7):3180-5.

Srivastava YK. Ecofriendly microwave assisted synthesis of some chalcones. Rasayan J, Chem, 2008; 1(4):884-6.

Straub TS. Epoxidation of $\alpha, \beta$-unsaturated ketones with sodium perborate. Tetrahedron Lett, 1995; 36(5):663-4.

Syahri J, Nasution H, Nurohmah BA, Purwono B, Yuanita E. Aminoalkylated chalcone: synthesis, biological evaluation, and docking simulation as potent antimalarial agents. J Appl Pharm Sci, 2020; 10(06):001-5.

Takahashi S, Kuroyama Y, Sonogashira K, Hagihara N. A convenient synthesis of ethynylarenes and diethynylarenes. Synthesis, $1980 ; 1980(08): 627-30$.

Venkataramireddy V, Shankaraiah V, Rao AT, Kalyani C, Narasu ML, Varala R, Jayashree A. Synthesis and anti-cancer activity of novel 3-aryl thiophene-2-carbaldehydes and their aryl/heteroaryl chalcone derivatives. Rasayan J Chem, 2016; 9(1):31-9.

Wan Z, Hu D, Li P, Xie D, Gan X. Synthesis, antiviral bioactivity of novel 4-thioquinazoline derivatives containing chalcone moiety. Molecules, 2015; 20(7):11861-74.

Wani ZA, Pathania AS, Mahajan G, Behl A, Mintoo MJ, Guru SK, Viswanath A, Malik F, Kamal A, Mondhe DM. Anticancer activity 
of a novel quinazolinone-chalcone derivative through cell cycle arrest in pancreatic cancer cell line. J Solid Tumors, 2015; 5(2):73-85.

Weindorf N, Schultz-Ehrenburg U. Controlled study of increasing venous tone in primary varicose veins by oral administration of Ruscus aculeatus and trimethylhespiridinchalcone. Z Hautkr, 1987; 62(1):28-38.

Won SJ, Liu CT, Tsao LT, Weng JR, Ko HH, Wang JP, Lin $\mathrm{CN}$. Synthetic chalcones as potential anti-inflammatory and cancer chemopreventive agents. Eur J Med Chem, 2005; 40(1):103-12.

Wu JZ, Cheng CC, Shen LL, Wang ZK, Wu SB, Li WL, Chen $\mathrm{SH}$, Zhou RP, Qiu PH. Synthetic chalcones with potent antioxidant ability on $\mathrm{H}_{2} \mathrm{O}_{2}$-induced apoptosis in PC12 cells. Int J Mol Sci, 2014; 15(10):18 $525-39$

Wu XF, Neumann H, Spannenberg A, Schulz T, Jiao H, Beller M. Development of a general palladium-catalyzedcarbonylative Heck reaction of aryl halides. J Am Chem Soc, 2010; 132(41):14596-602.
Xu LW, Li L, Xia CG, Zhao PQ. Efficient coupling reactions of arylalkynes and aldehydes leading to the synthesis of enones. Helv Chim Acta, 2004; 87(12):3080-4.

Zhang XW, Zhao DH, Quan YC, Sun LP, Yin XM, Guan LP. Synthesis and evaluate of anti-inflammatory activity of substituted chalcone derivatives. Med Chem Res, 2010; 19(4):403-12.

How to cite this article:

Goyal K, Kaur R, Goyal A, Awasthi R. Chalcones: A review on synthesis and pharmacological activities. J Appl Pharm Sci, 2021; 11 (Supp 1):001-014. 